\title{
Exposure to environmental tobacco smoke and sensitisation in children
}

\author{
E Lannerö, ${ }^{1,2} \mathrm{M}$ Wickman, ${ }^{1,3} \mathrm{M}$ van Hage, ${ }^{4}$ A Bergström, ${ }^{1} \mathrm{G}$ Pershagen, ${ }^{1,5}$ L Nordvall ${ }^{6}$
}

${ }^{1}$ Institute of Environmental Medicine, Karolinska Institutet, Stockholm, Sweden;

${ }^{2}$ Department of Paediatrics, Karolinska University Hospital, Huddinge, Sweden:

${ }^{3}$ Department of Paediatrics, Sach's Children's Hospital,

Stockholm, Sweden; ${ }^{4}$ Clinical Immunology and Allergy Unit, Department of Medicine, Karolinska Institutet and University Hospital, Stockholm, Sweden; ${ }^{5}$ Department of Occupational and Environmental Health, Stockholm County Council, Sweden; ${ }^{6}$ Department of Women's and Children's Health, Uppsala University, Uppsala, Sweden

Correspondence to: Dr E Lannerö, Karolinska University Hospital, Huddinge, 14186 Stockholm, Sweden; eva.lannero@ki.se

Received 1 February 2007 Accepted 2 September 2007

\section{ABSTRACT}

Background: Exposure to environmental tobacco smoke (ETS) increases the risk of respiratory illness in children but data are inconclusive regarding the risk of $\lg E$ sensitisation.

Objective: To elucidate whether exposure to smoking prenatally and/or postnatally is related to lgE sensitisation in children at 4 years of age.

Methods: As part of a prospective birth cohort study (BAMSE), a total of 4089 families with children answered questionnaires when the child was 2 months, 1, 2 and 4 years old on environmental factors and symptoms of allergic disease. Blood collected at age 4 years from 2614 children was analysed for $\lg E$ antibodies to common inhalant and food allergens. Odds ratios (OR) and 95\% confidence intervals (Cl) were calculated using logistic regression with adjustments for potential confounders.

Results: There was no evident association between maternal smoking during pregnancy and risk of $\lg \mathrm{E}$ sensitisation. In contrast, a dose-response effect was found for exposure to ETS from parental smoking during the first few months of life and lgE sensitisation. There was an increased risk of sensitisation to inhalant and/or food allergens $\left(\mathrm{OR}_{\text {adj }} 1.28(95 \% \mathrm{Cl} 1.01\right.$ to 1.62$\left.)\right)$ among children exposed to ETS at 2 months of age. The risk appeared particularly elevated for indoor inhalant allergens, such as cat $\left(\mathrm{OR}_{\mathrm{adj}} 1.96(95 \% \mathrm{Cl} 1.28\right.$ to 2.99$\left.)\right)$ and for food allergens $\left(\mathrm{OR}_{\text {adj }} 1.46(95 \% \mathrm{Cl} 1.11\right.$ to 1.93$\left.)\right)$. The IgE sensitising effect of ETS seemed to be confined to infants of parents without allergic diseases and to ETS exposure during early infancy.

Conclusions: Our data indicate that exposure in early infancy to ETS increases the risk of IgE sensitisation to indoor inhalant and food allergens.

The association between exposure to tobacco smoke in childhood and risk of atopic sensitisation has been extensively studied but data are inconclusive. In a comprehensive review in 1998, it was concluded that parental smoking is unlikely to increase the risk of IgE sensitisation in children. ${ }^{1}$ This review, however, primarily dealt with studies of relatively small size and none was a birth cohort study. Subsequent larger birth cohort studies still do not provide a consistent picture. Thus in the German Multicentre Allergy Study, no association was demonstrated between prenatal or postnatal exposure to tobacco smoke and IgE sensitisation to inhalant allergens at the 3 year follow-up whereas an association was seen for sensitisation to food allergens. ${ }^{2}$ In "The National Asthma Campaign in Manchester", little or no effect of environmental tobacco smoke (ETS) was found on the development of atopy.

We have previously reported a strong effect of cigarette smoke exposure in utero on recurrent wheezing in children up to 2 years of age in the BAMSE cohort. ${ }^{4}$ In the present study, we aimed to assess the separate roles of smoke exposure in utero and postnatally for IgE sensitisation to important inhalant and food allergens at 4 years of age.

\section{MATERIAL AND METHODS Study subjects}

A total of 4089 infants born in Stockholm during 1994-1996 were included in a prospective population based study (BAMSE). The cohort constituted $75 \%$ of all eligible children from the area. Details of the study design, inclusion criteria, enrolment and data collection procedure are described in detail elsewhere. ${ }^{5-7}$

\section{Exposure assessment}

At a median age of 2 months, the parents answered the first questionnaire, which aimed to collect information on exposure to environmental factors before and immediately after birth, such as exposure to tobacco smoke, and parental allergy. The socioeconomic status of the parents was assessed using questions about educational level and profession. ${ }^{8}$ Follow-up questionnaires on symptoms of disease as well as on exposure to ETS and other environmental factors were distributed at 1, 2 and 4 years of age. The response rate of the questionnaire at 4 years of age was $91 \%$. Maternal smoking during pregnancy was defined as smoking one cigarette or more per day during any trimester of pregnancy. Smoking was reported as number of cigarettes smoked for each trimester separately. ETS was defined as maternal and/or paternal smoking of one cigarette or more per day, when the child was 2 months, 1 or 2 years of age. In order to minimise bias from disease related misclassification of exposure, most analyses focused on ETS data in the 2 month questionnaire.

Exposure to locally emitted air pollution from traffic using nitrogen oxides as the indicator was assessed by retrospective methodology. ${ }^{9}{ }^{10}$ Emission databases within Stockholm County were available for nitrogen oxides from traffic in 1990 and 2000, and the outdoor levels were calculated using dispersion modelling by interpolation between these years.

\section{$\lg \mathrm{E}$ antibodies}

At 4 years of age, an invitation was sent for a clinical examination which included blood sampling. Samples were successfully obtained from 2614 of 2965 children whose parents agreed to participate in the examination. As a screening procedure, serum IgE antibodies to a mixture of inhalant (Phadiatop) and food ( $\mathrm{f} \times 5)$ allergens 
Table 1 Parental smoking* in relation to selected characteristics of children providing blood samples and the whole BAMSE cohort

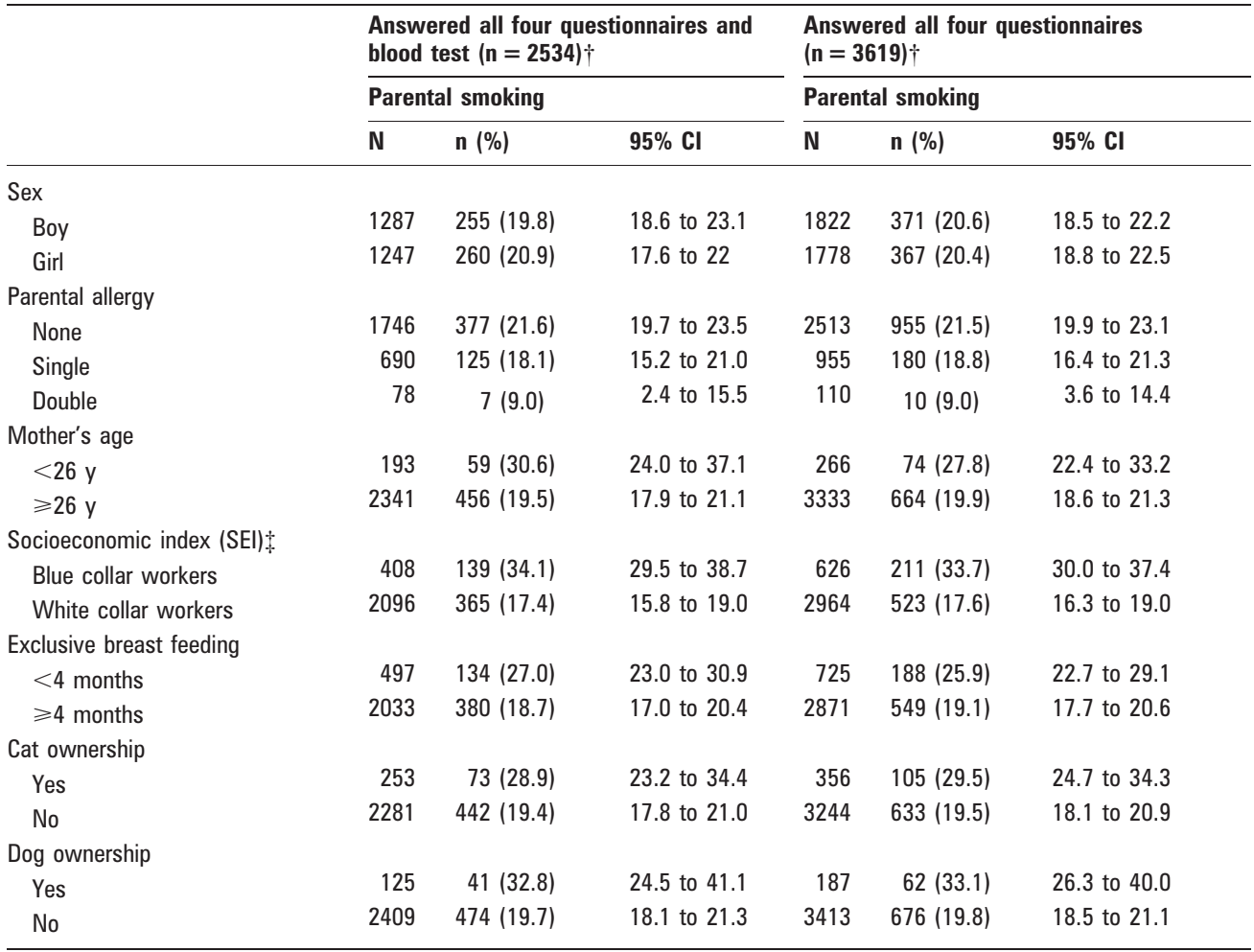

$\mathrm{N}$, total number in the group; $\mathrm{n}$, number exposed.

* Mother and/or father smoking at least one cigarette per day when the child was about 2 months old.

fInformation available on parental smoking when the child was about 2 months old.

$\$$ Socioeconomic status of the parents according to the socioeconomic index measured by the Nordic Standard Occupational

Classification (NYK) and Swedish Socioeconomic Classification (SEI).

(ImmunoCAP, Phadia AB, Uppsala, Sweden) were analysed according to the manufacturer's instructions. Sera that scored positive $\left(\geqslant 0.35 \mathrm{kU}_{\mathrm{A}} / \mathrm{l}\right)$ were further analysed for IgE antibodies to the respective individual allergen (birch, timothy, mugwort, cat, dog, horse, Dermatophagoides pteronyssinus, Cladosporium herbarum and cows milk, hen's egg, peanut, soy, wheat and cod fish). Only sera positive for at least one single allergen were considered positive. Any inhalant allergen refers to a positive test $\left(\geqslant 0.35 \mathrm{kU}_{\mathrm{A}} / \mathrm{l}\right)$ to birch, timothy, mugwort, Dermatophagoides pteronyssinus (dust mite), Cladosporium herbarum (mould), cat, dog and/or horse, and any food allergen to a positive test to any of the foods tested. Separate analyses were also performed using $3.5 \mathrm{kU} / 1$ as the cut-off.

\section{Statistical analyses}

The relation between parental smoking and sensitisation was analysed with unconditional logistic regression and the results presented as odds ratios (OR) and $95 \%$ confidence intervals ( $95 \%$ CI). Several models were tested, including various covariates. Models were tested which included potential confounders such as sex, parental allergy, socioeconomic status, maternal age, parental education, cat and dog ownership, duration of breastfeeding, older siblings, ambient NOx (nitrogen oxides from traffic) and reported signs of indoor dampness. In our final model, adjustments were made for parental allergic disease, defined as doctor's diagnosed asthma and asthma medication and/or allergic rhino-conjunctivitis in combination

Table 2 Exposure to maternal smoking during pregnancy or exposure to parental smoking when the infant was about 2 months old and sensitisation $\left(\lg E \geqslant 0.35 \mathrm{kU}_{\mathrm{A}} / \mathrm{l}\right)$ at 4 years of age

\begin{tabular}{|c|c|c|c|c|c|c|c|c|c|}
\hline & \multicolumn{3}{|c|}{ Any sensitisation*§ } & \multicolumn{3}{|c|}{ Any inhalant $\dagger \S$} & \multicolumn{3}{|c|}{ Any food $\$ \S$} \\
\hline & $\mathbf{N}$ & n & OR (95\% Cl) & N & n & OR (95\% Cl) & N & n & OR (95\% CI) \\
\hline No exposure in utero or ETS & 1917 & 399 & 1.00 (Referent) & 1917 & 273 & 1.00 (Referent) & 1917 & 228 & 1.00 (Referent) \\
\hline Exposure in utero but not to ETS & 98 & 21 & $1.00(0.61$ to 1.66$)$ & 98 & 15 & 1.05 (0.59 to 1.87$)$ & 98 & 15 & $1.28(0.72$ to 2.26$)$ \\
\hline Exposure to ETS but not in utero & 305 & 76 & $1.26(0.95$ to 1.68$)$ & 304 & 45 & $1.06(0.75$ to 1.50$)$ & 305 & 56 & 1.61 (1.16 to 2.24$)$ \\
\hline Exposure in utero and to ETS & 209 & 54 & $1.30(0.92$ to 1.82$)$ & 209 & 36 & 1.21 (0.81 to 1.81$)$ & 209 & 32 & $1.24(0.82$ to 1.89$)$ \\
\hline
\end{tabular}

\footnotetext{
$\mathrm{N}$, total number in the group; $\mathrm{n}$, number exposed.

${ }^{*}$ Any inhalant and/or any food allergen positive.

†Any inhalant (Phadiatop and at least one single allergen positive)

$\$$ Any food ( $\mathrm{f} \times 5$ and at least one single allergen positive).

§Adjusted for parental allergy and socioeconomic status.

ETS, environmental tobacco smoke.
} 
with reported allergy to furred pet and/or pollen in one parent (any heredity) or both (double heredity), and socioeconomic status since only these variables changed the OR estimates for tobacco smoke exposure by more than 3\%. Logistic regression was also used for trend analyses, and a $p$ value for the coefficient of $<0.05$ was considered statistically significant. Statistical analyses were performed with the Stata Statistical Software, release 8.0 (College Station, Texas, USA).

Only children whose parents answered all four questionnaires and had complete information on IgE sensitisation and ETS exposure at baseline (about 2 months of age) were included in the final analyses. This was true for 2534 children, or $62 \%$ of the original cohort.

The study was approved by the ethics committee at the Karolinska Institute, Stockholm, Sweden.

Table 3 Parental smoking at 2 months and risk of sensitisation (IgE $\left.\geqslant 0.35 \mathrm{kU}_{\mathrm{A}} / \mathrm{l}\right)$ to individual allergens at 4 years of age

\begin{tabular}{|c|c|c|c|}
\hline & $\mathbf{N}$ & 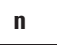 & $\mathbf{O R}_{\mathrm{adj}}{ }^{*}(95 \% \mathrm{Cl})$ \\
\hline \multicolumn{4}{|l|}{ Birch } \\
\hline No ETS & 2008 & 164 & 1.00 \\
\hline ETS & 508 & 57 & 1.25 (0.86 to 1.82$)$ \\
\hline \multicolumn{4}{|l|}{ Timothy } \\
\hline No ETS & 2010 & 116 & 1.00 \\
\hline ETS & 510 & 30 & $1.14(0.72$ to 1.81$)$ \\
\hline \multicolumn{4}{|l|}{ Mugwort } \\
\hline No ETS & 2006 & 33 & 1.00 \\
\hline ETS & 507 & 5 & $0.65(0.23$ to 1.81$)$ \\
\hline \multicolumn{4}{|l|}{ Cat } \\
\hline No ETS & 2017 & 97 & 1.00 \\
\hline ETS & 513 & 46 & 1.96 (1.28 to 2.99$)$ \\
\hline \multicolumn{4}{|l|}{ Dog } \\
\hline No ETS & 2013 & 79 & 1.00 \\
\hline ETS & 513 & 30 & $1.38(0.83$ to 2.30$)$ \\
\hline \multicolumn{4}{|l|}{ Horse } \\
\hline No ETS & 2012 & 49 & 1.00 \\
\hline ETS & 513 & 29 & 2.09 (1.21 to 3.63$)$ \\
\hline \multicolumn{4}{|l|}{ Dust mite } \\
\hline No ETS & 2013 & 33 & 1.00 \\
\hline ETS & 512 & 13 & $1.52(0.72$ to 3.23$)$ \\
\hline \multicolumn{4}{|l|}{ Mould } \\
\hline No ETS & 2007 & 13 & 1.00 \\
\hline ETS & 508 & 10 & $3.24(1.29$ to 8.12$)$ \\
\hline \multicolumn{4}{|l|}{ Cows milk } \\
\hline No ETS & 2015 & 157 & 1.00 \\
\hline ETS & 514 & 57 & $1.52(1.05$ to 2.18$)$ \\
\hline \multicolumn{4}{|l|}{ Peanut } \\
\hline No ETS & 2017 & 97 & 1.00 \\
\hline ETS & 511 & 38 & $1.54(0.99$ to 2.41$)$ \\
\hline \multicolumn{4}{|l|}{ Hen's egg } \\
\hline No ETS & 2016 & 91 & 1.00 \\
\hline ETS & 514 & 31 & 1.29 (0.80 to 2.09$)$ \\
\hline \multicolumn{4}{|l|}{ Wheat } \\
\hline No ETS & 2015 & 67 & 1.00 \\
\hline ETS & 511 & 28 & 1.37 (0.81 to 2.32$)$ \\
\hline \multicolumn{4}{|l|}{ Soybean } \\
\hline No ETS & 2017 & 55 & 1.00 \\
\hline ETS & 511 & 21 & $1.43(0.79$ to 2.57$)$ \\
\hline Cod fish† & & & \\
\hline
\end{tabular}

$\mathrm{N}$, total number in the group; $\mathrm{n}$, number exposed.

${ }^{*}$ Adjusted for parental allergy, defined as asthma and/or allergic rhino-conjunctivitis diagnosed by a doctor and in combination with reported allergy to furred pets and/or pollen in one or both parents (reported asthma medication was required for asthma diagnosis), maternal smoking during pregnancy and socioeconomic status.

$\uparrow$ Too few observations to perform multivariate analysis.

ETS, environmental tobacco smoke.

\section{RESULTS}

Table 1 presents parental smoking when the child was 2 months old in relation to some important exposure characteristics among those who had answered all four questionnaires and provided a blood sample (ie, those eligible for this study) as well as for those representing the whole dataset (ie, those who answered all four questionnaires). There were no significant differences between these two groups. Among the 2534 study participants, $12 \%$ of the mothers reported smoking one cigarette or more daily during one or two trimesters of pregnancy and $8 \%$ smoked all through pregnancy. At 2 months of age, $20 \%$ of children were exposed to ETS from any parent, including $4 \%$ exposed to smoking by both parents. Participating children with heredity allergic disease were significantly less exposed to parental smoking than children without such heredity $(p=0.007)$. Parental smoking was also associated with low maternal age, low socioeconomic status, cat and dog ownership and short duration of breast feeding.

At 4 years of age, $24 \%$ of the children were $\operatorname{IgE}$ sensitised, $15 \%$ to inhalant allergens, $16 \%$ to food allergens and $7 \%$ to both. There was no evident increase in the risk of any sensitisation (ie, inhalant and/or food allergens) if the mother had smoked during any of the trimesters ( $\left(\mathrm{R}_{\mathrm{adj}} 1.00\right.$ (95\% CI 0.61 to 1.66)) but not thereafter (table 2) On the other hand, exposure to ETS at 2 months of age without previous in utero exposure tended to be associated with sensitisation $\left(\mathrm{OR}_{\mathrm{adj}} 1.26\right.$ (95\% CI 0.95 to 1.68)) and there was no clear evidence of interaction between in utero and postnatal exposure. For postnatal ETS exposure, with or without exposure in utero, the adjusted ORs for sensitisation to inhalant allergens were 1.12 (95\% CI 0.84 to 1.48), for food allergens 1.46 (95\% CI 1.11 to 1.93$)$ and for any sensitisation 1.28 (95\% CI 1.01 to 1.62$)$.

In table 3 , the results on ETS and sensitisation to the most important single allergens are presented. Exposure to ETS was associated with increased risks of sensitisation to cat, horse and mould as well as to food allergens (eg, milk and peanuts). In contrast, no clear association was evident with respect to seasonal outdoor allergens (ie, birch, timothy and mugwort).

Separate analyses of sensitisation using a cut-off value of $>3.5 \mathrm{kU}$ A $/ 1$ of $\mathrm{IgE}$ antibody were also performed. Exposure to ETS at 2 months of age resulted in an adjusted OR of 1.26 (95\% CI 0.86 to 1.84) for sensitisation to inhalant and/or food allergens. Only sensitisation to cat among single allergens showed a statistically significant increase in risk using the higher cut-off value $\left(\mathrm{OR}_{\mathrm{adj}} 2.59\right.$ (95\% CI 1.40 to 4.78$)$ ) but the power was low for most other single allergens.

Furthermore, a dose-response effect was found for the association between exposure of ETS at 2 months of age and IgE sensitisation (table 4). Thus when testing for trend (no, one, both parents smoke one cigarette or more daily and both parents smoke $>10$ cigarettes a day) a significant relationship was found $\left(p_{\text {trend }}=0.019\right)$. A strong trend was shown for sensitisation to food allergens ( $p_{\text {trend }}=0.006$ ) but not for inhalant allergens $\left(p_{\text {trend }}=0.496\right)$. Among single allergens, the strongest trend was seen for sensitisation to cat $\left(p_{\text {trend }}=0.003\right)$. A trend was also found for the association between paternal, maternal or biparental smoking and $\operatorname{IgE}$ sensitisation $\left(\mathrm{OR}_{\mathrm{adj}}\right.$ 1.21 (95\% CI 0.91 to 1.61$), \mathrm{OR}_{\text {adj }} 1.24$ (95\% CI 0.68 to 2.24$)$ and $\mathrm{OR}_{\text {adj }} 1.87$ (95\% CI 1.06 to 3.31), respectively; $\left.p_{\text {trend }}=0.024\right)$. In stratified analyses, the main interaction was seen with parental allergy, where the effect of ETS exposure seemed stronger among children with non-allergic parents $\left(\mathrm{OR}_{\mathrm{adj}} 1.95\right.$ (95\% CI 1.19 to 3.20$)$ ) than among those with allergic parents $\left(\mathrm{OR}_{\mathrm{adj}}\right.$ 1.06 (95\% CI 0.47 to 2.35$)$ ). 
Table 4 Exposure to parental cigarette smoking at 2 months and sensitisation $\left(\lg E \geqslant 0.35 \mathrm{kU}_{\mathrm{A}} / \mathrm{l}\right)$ to any inhalant and/or food allergen at 4 years of age

\begin{tabular}{|c|c|c|c|c|c|}
\hline & $\mathbf{N}$ & $\mathbf{n}$ & $\begin{array}{l}\text { Crude model } \\
\text { OR }(95 \% \text { Cl) }\end{array}$ & $\begin{array}{l}\text { Adjusted for parental allergy } \\
\text { and socioeconomic status } \\
\text { OR }(95 \% \mathrm{CI})\end{array}$ & $\begin{array}{l}\text { Additional adjustment for } \\
\text { maternal smoking during } \\
\text { pregnancy } \\
\text { OR }(95 \% \mathrm{Cl})\end{array}$ \\
\hline Only one parent smokes ( $\geqslant 1$ cigarette a day) & 408 & 99 & 1.19 (0.93 to 1.53$)$ & $1.19(0.92$ to 1.54$)$ & $1.21(0.93$ to 1.59$)$ \\
\hline $\begin{array}{l}\text { Both parents smoke (one or both less than } \\
10 \text { cig a day) }\end{array}$ & 59 & 17 & $1.54(0.87$ to 2.73$)$ & $1.68(0.94$ to 2.99$)$ & $1.79(0.94$ to 3.38$)$ \\
\hline Both parents smoke $\geqslant 10$ cig a day & 31 & 11 & $1.82(0.88$ to 3.76$)$ & 1.84 (0.89 to 3.84$)$ & $1.99(0.89$ to 4.45$)$ \\
\hline
\end{tabular}

$\mathrm{N}$, total number in the group; $n$, number exposed.

In extended analyses, the role of ETS exposure after 2 months was also assessed (table 5). The strongest association between exposure to ETS and any sensitisation (any inhalant allergen and/or any food allergen) appeared for exposure at 2 months but not thereafter $\left(\mathrm{OR}_{\mathrm{adj}} 1.63\right.$ (95\% CI 1.07 to 2.49$)$ ). If anything, a decreased risk was suggested among those exposed to ETS at 1 and 2 years only.

\section{DISCUSSION}

It is evident that exposure to smoke in early life, most particularly exposure of the fetus, causes wheezing in infants. ${ }^{41-13}$ However, with regard to atopic sensitisation, the evidence for an effect of smoke exposure has been equivocal. ${ }^{1-3}$ The present study provides evidence indicating that exposure in early infancy to tobacco smoke may be associated with an increased risk of atopic sensitisation. The finding of dose-response relationships supports the interpretation of causality. Furthermore, there was also an effect of ETS on IgE antibody responses above $3.5 \mathrm{kU} / \mathrm{A}$, which suggests that the findings could be of clinical importance. Some reasons for the apparent difference in results compared with previous studies may be the considerably larger size of our study and separation of the effect of exposure to maternal smoking during pregnancy from that of ETS as well as differentiation of sensitisation to indoor perennial and outdoor seasonal allergens. The associations were particularly strong with respect to cat allergen, the most important perennial allergen in our population. It is well known both from clinical experience and studies that exposure to cats leads to more symptoms than exposure to dogs. ${ }^{14}$ Cat allergen is ubiquitous indoors in our part of the world both in homes and in schools, which contributes to the clinical importance of cat allergy. ${ }^{15} 16$

It was difficult to fully disentangle the effects of exposure in utero and postnatally in our study because of a high correlation between the two types of exposure. However, no consistent effects on sensitisation were seen among children exposed only prenatally. As regards exposure to ETS, we have relied primarily on the information on exposure collected in the first questionnaire at 2 months of age in order to ensure the prospective nature of data and avoid any bias resulting from modification or misreporting of smoking habits as a consequence of symptoms in the child. Such disease related bias would tend to reverse the association between ETS and sensitisation/allergy, as suggested in our data, where the risk of sensitisation tended to be inversely related to reported parental smoking at 1 and 2 years of age. In Sweden, virtually all mothers attend maternity health clinics and the awareness of the detrimental effects of smoking is very high. Avoidance measures following debut of allergic symptoms in children have been documented in our cohort. ${ }^{17} \mathrm{~A}$ contributing explanation as to why the sensitising effect of ETS appears to be confined to exposure during the first few months of life could well be that smoke exposure of infants from their parents is significantly reduced at the end of the first year of life and later, as most children in Sweden start daycare outside their homes at around 1 year of age. Thus by 2 years of age, more than $75 \%$ of the children in the BAMSE cohort were in daycare outside their homes. Furthermore, exposure during the first months of life may be particularly detrimental for induction of sensitisation, as indicated for exposure to pollen ${ }^{18} 19$ and ambient air pollution. ${ }^{20}$ The lack of focus on a potentially important time window for sensitisation and disease related misclassification of exposure may have contributed to the negative findings in previous studies on ETS exposure and sensitisation. It is also notable that the effect of early exposure to ETS was primarily confined to children without parental allergy, which may partly be attributable to their parent's significantly higher degree of smoking.

Our data also raise the more general issue of effects on sensitisation by other air pollutants, such as traffic related air pollution. There appears to be a relationship between ambient air pollution and wheezing/asthma in children. ${ }^{21-25}$ With regard to IgE sensitisation, the literature is more ambiguous but several studies $^{20}{ }^{24-27}$ indicate a relationship between traffic related air pollution and atopy. These findings and our data support the "mucosal concept of atopy", which implies that sensitisation occurs mainly in the mucosal surfaces of the airways and that mucosal damage and inflammation facilitate sensitisation. ${ }^{28}$ It is

Table 5 Exposure to parental cigarette smoking at different ages and sensitisation ( $\left.\lg E \geqslant 0.35 \mathrm{kU}_{\mathrm{A}} / \mathrm{l}\right)$ to any inhalant and/or food allergen at 4 years of age

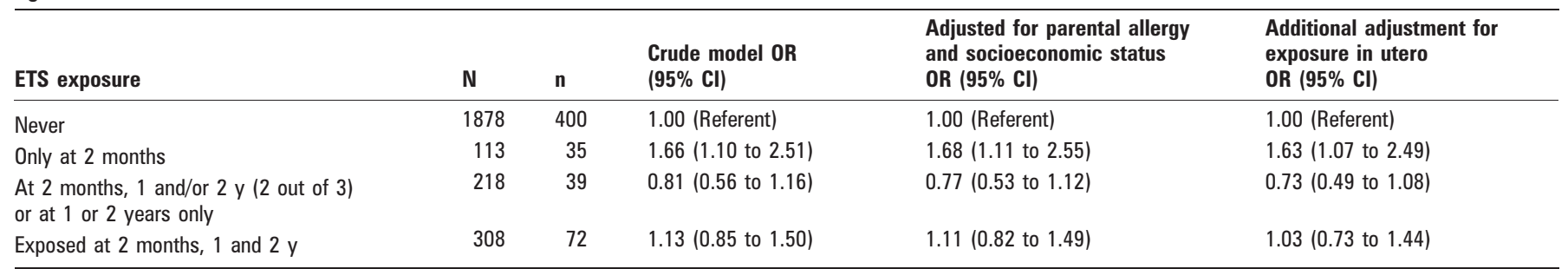

ETS, environmental tobacco smoke; N, total number in the group; n, number exposed. 
noteworthy that smoke exposure appeared to facilitate sensitisation to the perennial indoor allergens, such as cat, and not to the same extent sensitisation to seasonal pollen allergens (birch, timothy and mugwort) to which we are mainly exposed outdoors where the inhaled dose of tobacco smoke exposure is limited. We also found an increased risk of sensitisation to common foods with exposure to ETS, which supports earlier findings. ${ }^{2}$ It may seem puzzling that ETS should increase the risk of sensitisation to food allergens. Such allergens are, however, likely to be found in house dust. Swallowed foods are probably also to some extent inhaled or aspirated by a baby, and is why sensitisation appears a conceivable consequence, which could be facilitated by tobacco smoke exposure. Other possibilities are that exposure to the pharynx and pharyngeal lymphoid tissue both of food and inhalant allergen as well as tobacco smoke may be of importance and/or such an influence on the airway and/or pharyngeal epithelium causes a more profound disturbance of the immune system.

Some methodological issues deserve consideration. Since the assessment of exposure to tobacco smoke was done by parental questionnaire based reports, an obvious disadvantage in our design is the lack of objective smoke exposure assessments. However, a strong correlation has been found between assessment of ETS exposure using urinary cotinine and selfreported data. ${ }^{29}{ }^{30}$ Data on maternal smoking during pregnancy were collected when the child was about 2 months old. Thus, particularly when it comes to these data, there could be a risk of recall bias, but validation studies indicate that this is not pronounced. ${ }^{3}$ The potential for selection bias has to be considered. Such bias may be introduced by non-participation due to parental awareness of the hazardous effects of cigarette smoking. Consequently, smokers may to a higher extent than non-smokers have chosen not to participate in the study. A study of non-responders and excluded families of the BAMSE study showed that non-participating parents smoked more than those recruited to the study. ${ }^{5}$ The study base may thus be less representative of the population, but the risk estimates of health effects related to smoking would most likely not be affected. Another possibility is that parents with allergic diseases would be more willing to join the study compared with parents without such diseases, but no such evidence was found. ${ }^{5}$ Several potential confounding factors were assessed but only parental allergy and socioeconomic status had any clear effect on the risk estimates. As the study is rich in information on risk factors for allergy and sensitisation in children, which have been extensively assessed and analysed, it is unlikely that uncontrolled confounding is of importance for our findings.

In conclusion, our data indicate that ETS exposure in early infancy increases the risk of sensitisation to indoor inhalant and food allergens. The effects were consistent and strong, and further supported by positive dose-response relationships. These effects may be of great clinical relevance as sensitisation is important for incident asthma among children and adults.

\begin{abstract}
Acknowledgements: Thanks to all colleagues at the Department of Occupational and Environmental Health, Stockholm County Council, Stockholm, Sweden who worked with the BAMSE project. Also thanks to the children and their parents for participating in the BAMSE study.
\end{abstract}

Funding: This study was funded by the Swedish Heart and Lung Foundation, Stockholm County Council, the Gillbergska Foundation and the Swedish Asthma and Allergy Foundation, The Swedish Foundation for Health Care Sciences and Allergy Research (Vårdalstiftelsen) and The Swedish Research Council.

Competing interests: None.

\section{REFERENCES}

1. Strachan DP, Cook DG. Health effects of passive smoking .5. Parental smoking and allergic sensitisation in children. Thorax 1998;53:117-23.

2. Kulig M, Luck W, Lau S, et al. Effect of pre- and postnatal tobacco smoke exposure on specific sensitization to food and inhalant allergens during the first 3 years of life. Multicenter Allergy Study Group, Germany. Allergy 1999;54:220-8.

3. Murray CS, Woodcock A, Smillie Fl, et al. Tobacco smoke exposure, wheeze, and atopy. Pediatr Pulmonol 2004;37:492-8.

4. Lannerö $\mathbf{E}$, Wickman M, Pershagen G, et al. Maternal smoking during pregnancy increases the risk of recurrent wheezing during the first years of life (BAMSE). Respir Res 2006;7:3

5. Wickman M, Kull I, Pershagen G, et al. The BAMSE Project: presentation of a prospective longitudinal birth cohort study. Pediatr Allergy Immunol 2002;13/Suppl 15):11-13.

6. Lannerö E, Kull I, Wickman M, et al. Environmental risk factors for allergy and socioeconomic status in a birth cohort (BAMSE). Pediatr Allergy Immunol 2002;13:182-7.

7. Kull I, Wickman M, Lilja G, et al. Breastfeeding and allergic diseases in infants-a prospective birth cohort study. Arch Dis Child 2002;87:478-81.

8. Hallqvist $\mathbf{J}$, Lundberg $M$, Diderichsen $F$, et al. Socioeconomic differences in risk of myocardial infarction 1971-1994 in Sweden: time trends, relative risks and population attributable risks. Int J Epidemiol 1998;27:410-15.

9. Bellander T, Berlind N, Gustavsson P, et al. Using geographic information systems to assess individual historical exposure to air pollution from traffic and house heating in Stockholm. Environ Health Perspect 2001;109:633-9.

10. Rosenlund $\mathbf{M}$, Berglind $\mathrm{N}$, Hallqvist J, et al. Long-term exposure to urban air pollution and myocardial infarction. Epidemiology 2006;17:383-90.

11. Strachan DP, Cook DG. Health effects of passive smoking. 1. Parental smoking and lower respiratory illness in infancy and early childhood. Thorax 1997;52:905-14.

12. Lux AL, Henderson AJ, Pocock SJ. Wheeze associated with prenatal tobacco smoke exposure: a prospective, longitudinal study. ALSPAC Study Team. Arch Dis Child 2000;83:307-12

13. Wickman M, Nordvall SL, Pershagen G. Risk factors in early childhood for sensitization to airborne allergens. Pediatr Allergy Immunol 1992;3:128-33.

14. Murray AB, Ferguson AC, Morrison BJ. The frequency and severity of cat allergy vs dog allergy in atopic children. J Allergy Clin Immunol 1983;72:145-9.

15. Munir AK, Einarsson R, Dreborg S. Variability of airborne cat allergen, Fel d1, in a public place. Indoor Air 2003;13:353-8.

16. Almqvist C, Larsson PH, Egmar AC, et al. School as a risk environment for children allergic to cats and a site for transfer of cat allergen to homes. J Allergy Clin Immunol 1999;103:1012-17.

17. Kull I, Bergstrom A, Lilja G. Fish consumption during the first year of life and development of allergic diseases during childhood. Allergy 2006;61:1009-15.

18. Bjorksten F, Suoniemi I. Dependence of immediate hypersensitivity on the month of birth. Clin Allergy 1976:6:165-71.

19. Kihlstrom A, Lilja G, Pershagen G, et al. Exposure to birch pollen in infancy and development of atopic disease in childhood. J Allergy Clin Immunol 2002;110:78-84.

20. Nordling $\mathbf{E}$, Berglind $\mathrm{N}$, Melén $\mathrm{E}$, et al. Exposure to traffic-related air pollution in early life affects airway disease, lung function and allergic sensitization in 4-year old children. 2007 (under review following revision).

21. Brauer $\mathbf{M}$, Hoek $G$, Van Vliet $P$, et al. Air pollution from traffic and the development of respiratory infections and asthmatic and allergic symptoms in children. Am J Respir Crit Care Med 2002;166:1092-8.

22. Pierse N, Rushton L, Harris RS, et al. Locally generated particulate pollution and respiratory symptoms in young children. Thorax 2006;61:216-20.

23. Nicolai T, Carr D, Weiland SK, et al. Urban traffic and pollutant exposure related to respiratory outcomes and atopy in a large sample of children. Eur Respir J 2003;21:956-63

24. Wyler C, Braun-Fahrländer C, Künzli N, et al. Exposure to motor vehicle traffic and allergic sensitization. The Swiss Study on Air Pollution and Lung Diseases in Adults (SAPALDIA) Team. Epidemiology 2000;11:450-6.

25. Pénard-Morand C, Charpin D, Raherison C, et al. Long-term exposure to background air pollution related to respiratory and allergic health in schoolchildren. Clin Exp Allergy 2005;35:1279-87.

26. Krämer U, Koch T, Ranft U, et al. Traffic-related air pollution is associated with atopy in children living in urban areas. Epidemiology 2000;11:64-70.

27. Janssen NA, Brunekreef $B$, van Vliet $P$, et al. The relationship between air pollution from heavy traffic and allergic sensitization, bronchial hyperresponsiveness, and respiratory symptoms in Dutch schoolchildren. Environ Health Perspect 2003;111:1512-18.

28. Leskowitz S, Salvaggio JE, Schwartz HJ. An hypothesis for the development of atopic allergy in man. Clin Allergy 1972:2:237-46.

29. Brunekreef B, Leaderer BP, van Strien R, et al. Using nicotine measurements and parental reports to assess indoor air: the PIAMA birth cohort study. Prevention and Incidence of Asthma and Mite Allergy. Epidemiology 2000;11:350-2.

30. Willers S, Axmon A, Feyerabend C, et al. Assessment of environmental tobacco smoke exposure in children with asthmatic symptoms by questionnaire and cotinine concentrations in plasma, saliva, and urine. J Clin Epidemiol 2000;53:715-21. 